\title{
Probing the excitation spectrum of a Fermi gas in the BCS-BEC crossover regime
}

\author{
M. Greiner * C. A. Regal, and D. S. Jin \\ JILA, National Institute of Standards and Technology and University of Colorado, \\ and Department of Physics, University of Colorado, Boulder, CO 80309-0440
}

(Dated: October 9, 2018)

\begin{abstract}
We measure excitation spectra of an ultracold gas of fermionic ${ }^{40} \mathrm{~K}$ atoms in the BCS-BEC crossover regime. The measurements are performed with a novel spectroscopy that employs a small modulation of the B-field close to a Feshbach resonance to give rise to a modulation of the interaction strength. With this method we observe both a collective excitation as well as the dissociation of fermionic atom pairs in the strongly interacting regime. The excitation spectra reveal the binding energy / excitation gap for pairs in the crossover region.
\end{abstract}

The predicted crossover between Bardeen-CooperSchrieffer (BCS) type superfluidity and Bose-Einstein condensation (BEC) of molecules [1, 2, 3, 4, 5, 6, 7] has recently become experimentally accessible with a strongly interacting gas of ultracold fermionic atoms 8, 19, 10, 11, 12, 13, 14, 15, 16, 17. The atom-atom interactions in such a gas, characterized by the $s$-wave scattering length $a$, can be widely tuned with a magneticfield Feshbach resonance 18, 19, 20]. On the BEC side of the Feshbach resonance, the interactions are strong and repulsive $(a>0)$, and there exists a weakly bound molecular state. Bose-Einstein condensation of molecules in this state has been observed [8, 9, 10, 11]. On the BCS side of the resonance the gas has strong attractive interactions $(a<0)$. While no two-body bound molecular state exists in this region, many-body effects can give rise to pairing. Condensates of these pairs have recently been observed in gases of ${ }^{40} \mathrm{~K}\left[13\right.$ ] and ${ }^{6} \mathrm{Li}$ atoms 14 . The wide Feshbach resonances used in these experiments arise from strong coupling between open and closed scattering channels; therefore these gases are expected to be well described by BCS-BEC crossover physics 21] (see also 22] and references therein).

To probe the many-body state and the nature of the pairs, the excitation spectrum of the gas can be measured. Measurements of collective excitations, for example, have provided further evidence for superfluidity in the crossover regime [16, 17]. To probe single (quasi-) particle excitations, rf spectroscopy can be performed by applying a radio-frequency (rf) field and inducing transitions to different Zeeman or hyperfine levels. This rf spectroscopy has been used to measure mean-field shifts and interaction strengths 23, 24. In addition, on the BEC side of the Feshbach resonance rf excitation was used to measure the dissociation spectra and binding energies of weakly bound molecules 25]. Recently, this technique has been applied to measure the pairing gap in the BCSBEC crossover regime [26, 27, 28].

Here we report on a new spectroscopy that takes advantage of the tunability of interactions near a Feshbach resonance and probes the excitation spectrum in the BCS-BEC crossover region. We excite the system by modulating the interaction strength in the gas; this is accomplished using a small sinusoidal modulation of the $B$-field close to the Feshbach resonance. We find that the $B$-field modulation can cause a dissociation of bound molecules or generalized Cooper pairs into the free atom continuum. In contrast to rf spectroscopy this method does not involve additional spin states, but rather probes the excitation spectrum directly with no frequency shifts due to the properties of additional spin states. Also unlike rf spectroscopy, the $B$-field modulation does not drive single-atom excitations.

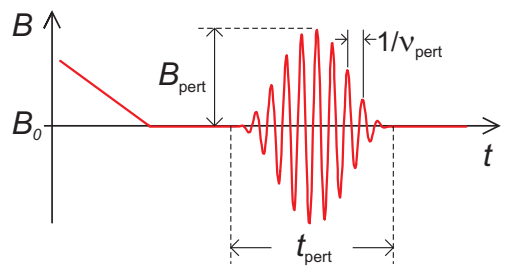

FIG. 1: Modulation sequence: After ramping to the final magnetic-field value $B_{0}$, the magnetic field is sinusoidally modulated at a frequency $\nu_{\text {pert. }}$. The envelope of the modulation is a haversine function with a maximum amplitude $B_{\text {pert }}$ and a total duration $t_{\text {pert }}$.

The experimental setup and procedure are similar to that in our previous work 23, 29]. We trap and cool a dilute gas of the fermionic isotope ${ }^{40} \mathrm{~K}$, which has a total spin $f=9 / 2$ in its lowest hyperfine ground state and thus ten available Zeeman spin states $\left|f, m_{f}\right\rangle$. The experiment is initiated by preparing a nearly equal, incoherent mixture of the $|9 / 2,-9 / 2\rangle$ and $|9 / 2,-7 / 2\rangle$ spin states. The atoms, as well as the molecules we create from these atoms, are trapped in a far off-resonant optical dipole trap. The trap is formed by a gaussian laser beam at a wavelength of $\lambda=1064 \mathrm{~nm}$ focused to a waist of $15.2 \mu \mathrm{m}$. We evaporatively cool the atoms to ultralow temperatures by gradually decreasing the trap depth. Our calculated final trap depth, including gravitational sag, is $h \times 9.6 \mathrm{kHz}$, where $h$ is Planck's constant. The measured Fermi energy in this trap is $h \times 4.6 \pm 0.6 \mathrm{kHz}$ 30] in the weakly interacting regime. The calculated radial trapping frequency in the center of the gaussian pro- 
file is $\nu_{r}=345 \mathrm{~Hz}$. We measure an effective radial trap frequency of $243 \mathrm{~Hz}$ for the weakly interacting gas; this is consistent with the trap frequency expected for a particle at about the Fermi energy in the non-harmonic trapping potential. At the final trap depth the optical trap holds $N_{0}=(1.8 \pm 0.9) \times 10^{5}$ atoms per spin state and the temperature of the weakly interacting gas, determined by fits to the cloud in expansion, is $T / T_{F}=0.05 \pm 0.02$.

The interaction between atoms in the two spin states is widely tunable with an $s$-wave magnetic-field Feshbach resonance, which is located at $202.10 \pm 0.07 \mathrm{G}[13$ and has a width of $7.8 \pm 0.6 \mathrm{G}$ 31]. Initially we prepare a quantum degenerate Fermi gas in the weakly interacting region far on the BCS side $(\Delta B>0)$ of the Feshbach resonance. Then we reduce the magnetic field adiabatically to bring the gas isentropically into the region of strong attractive atom-atom interactions. For B-fields up to $500 \mathrm{mG}$ away from the resonance on the BCS side we observe a condensate of pairs of fermionic atoms [13]. These pairs are attributable to many-body effects and can be regarded as generalized Cooper pairs in the BCS-BEC crossover regime.

We measure the excitation spectrum of the gas at different final magnetic field values $B_{0}$ by perturbatively modulating the magnetic field around $B_{0}$ with a frequency $\nu_{\text {pert }}$ (fig. (1). This sinusoidal $B$-field modulation causes a modulation of the effective atom-atom interaction strength. Starting at $t=0$ we modulate the B-field for a total duration $t_{\text {pert }}$ between $5 \mathrm{~ms}$ and $20 \mathrm{~ms}$. The modulation envelope is given by a haversine function with a maximum amplitude $B_{\text {pert }}$ (see fig. 1). In order to keep the response perturbative, we scaled the applied modulation amplitude as $B_{\text {pert }}=b_{0} / \nu_{\text {pert }}^{1 / 2}$, where $b_{0}$ ranges between $75 \mathrm{mG} \mathrm{kHz}^{1 / 2}$ close to the resonance and $220 \mathrm{mG} \mathrm{kHz}^{1 / 2}$ far from the Feshbach resonance.

To quantify the response of the gas, we determine the increase in the gas temperature after the perturbation is applied. We have found that a sensitive way to detect this increase in temperature is to measure the number of atoms that escape from the shallow trap due to evaporation. The evaporated atoms are accelerated downwards due to gravity and form a beam of atoms leaving the trap that can be observed by resonant absorption imaging. By fitting the absorption images to an empirical function we measure the number of atoms, $N_{\text {evap }}$, that are evaporated in the time between the start of the modulation and $4 \mathrm{~ms}$ after the modulation:

$$
N_{\text {evap }}=\int_{0}^{t_{\text {pert }}+4 \mathrm{~ms}} \dot{N}(t) d t
$$

Figure 2 shows measured excitation spectra for different interparticle interactions. The relative number of evaporated atoms $\left(N_{\text {evap }}-N_{b k g}\right) / N_{0}$, which provides a measure of the excitation response, is plotted versus the

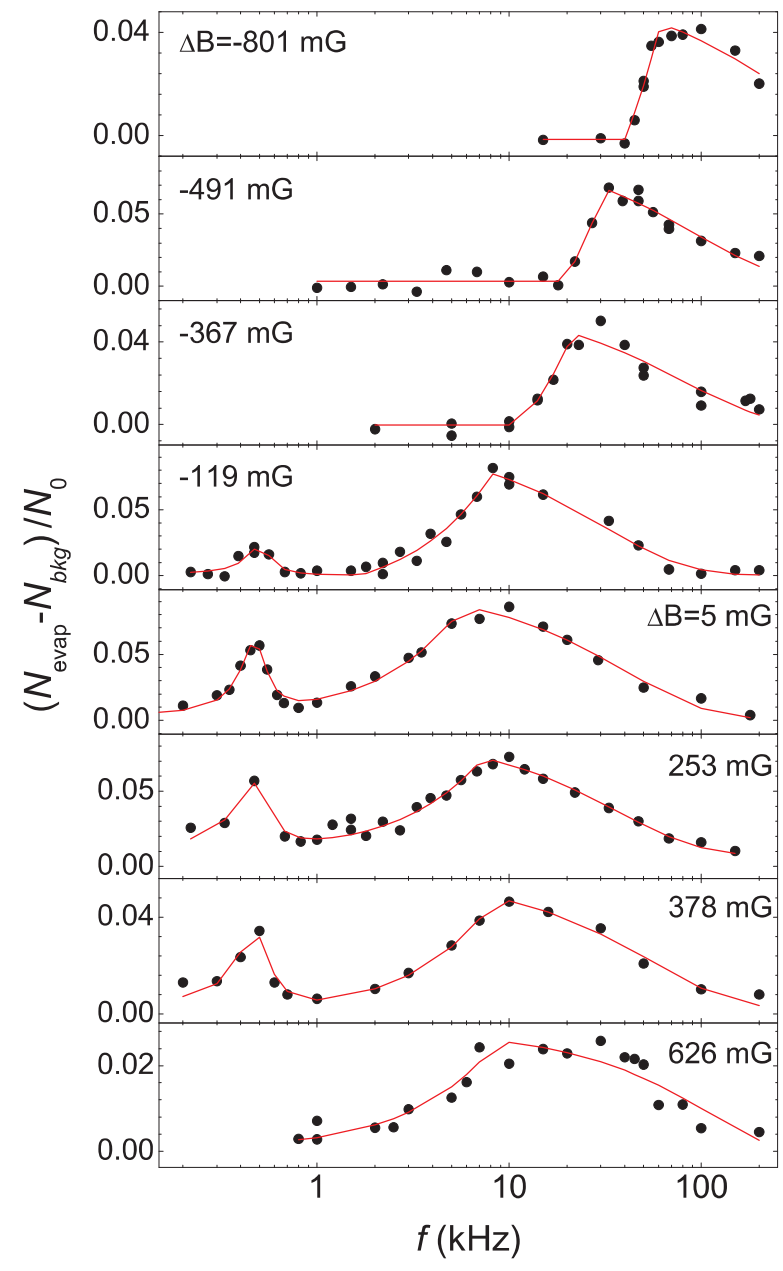

FIG. 2: Excitation spectra for different detunings $\Delta B=$ $B_{0}-202.10 \mathrm{G}$ with respect to the Feshbach resonance. The relative number of evaporated atoms $\left(N_{\text {evap }}-N_{b k g}\right) / N_{0}$ is a measure of the response and is plotted versus the modulation frequency $\nu_{\text {pert }}$. The modulation amplitude was scaled as $B_{\text {pert }}=b_{0} / \nu_{\text {pert }}^{1 / 2}$ to keep the response perturbative. The line is a fit to an empirical function, from which we can extract the threshold position $\nu_{0}$ and the position $\nu_{\max }$ and height $\tilde{N}_{\max }$ of the maximum.

frequency of the perturbation $\nu_{\text {pert }}$ on a logarithmic scale. Here, $N_{b k g}$ is the small number of evaporated atoms observed with no applied perturbation. The following features can be seen:

- We find a distinct peak in the excitation spectra for modulation frequencies $\nu_{\text {pert }} \approx 500 \mathrm{~Hz}$, which is close to twice the trap frequency. We attribute this peak to a collective excitation of the trapped gas driven by the periodic modulation of the interaction strength.

- For frequencies larger than a threshold $\nu_{0}$ the response increases. We interpret this threshold as a dissociation threshold: Pairs are only dissociated 
if $h \nu_{\text {pert }}$ is larger than the effective pair binding energy. On the BEC side of the resonance $\nu_{0}$ is nonzero and increases for decreasing $B_{0}$, consistent with two-body predictions for molecule binding energies.

- For increasing frequency beyond $\nu_{0}$ the response reaches a maximum and then slowly decreases.

We have measured the dependence of the dissociation response of the gas on the amplitude and duration of the B-field modulation. The number of evaporated atoms depends linearly on the duration and quadratically on the amplitude of the modulation, with a small offset due to a constant background heating and evaporation rate. The response due to the dissociation of pairs can be understood as follows: After dissociation the two free atoms gain a total relative kinetic energy $\Delta E=h \nu_{\text {pert }}-E_{b}$, where $E_{b}$ is the effective pair binding-energy or twice the excitation gap and $h$ is Planck's constant. Since the sample is collisionally dense in the region of strong interactions close to the Feshbach resonance, the dissociated atoms undergo multiple collisions and deposit this energy into the gas. We have verified that the measured response, $\left(N_{\text {evap }}-N_{b k g}\right) / N_{0}$, is proportional to the heating measured directly in a deeper trap. The spectra in fig. 2 are then proportional to

$$
\Gamma(\nu) \frac{h \nu_{\text {pert }}-E_{b}}{h \nu_{\text {pert }}},
$$

where $\Gamma(\nu)$ is the frequency dependent dissociation rate and the factor $\left(h \nu_{\text {pert }}\right)^{-1}$ accounts for the modulation amplitude being scaled as $\left(h \nu_{\text {pert }}\right)^{-1 / 2}$.

For a quantitative study we have fit the data to an empirical model (line in fig. 2). The collective excitation peak at low modulation frequencies is fit with a Lorentz curve. The subsequent increase of the response is fit to a linear slope, starting at the threshold $\nu_{0}$ (note that the linear curve appears bent in the logarithmic plot). After the maximum, the fit function consists of an exponential decay.

Figure 3 shows the positions of the fitted threshold $\nu_{0}$ and the response maximum $\nu_{\max }$ versus the B-field relative to the Feshbach resonance position, $\Delta B$. For comparison we also show a plot of the two-body binding energy in units of Hertz from a full coupled channel calculation 32] on the BEC side of the Feshbach resonance. The measured threshold for dissociation is close to the two-body binding energy of the molecules. The data might suggest that the actual position of the Feshbach resonance is at slightly higher magnetic fields, closer to the upper limit $(+70 \mathrm{mG})$ given in ref. [13] (dotted line). However, the data in fig. 3 are measured at large densities and strong interaction strengths where mean-field shifts, arising from the differences between molecule and atom interactions, are expected to play a significant role.

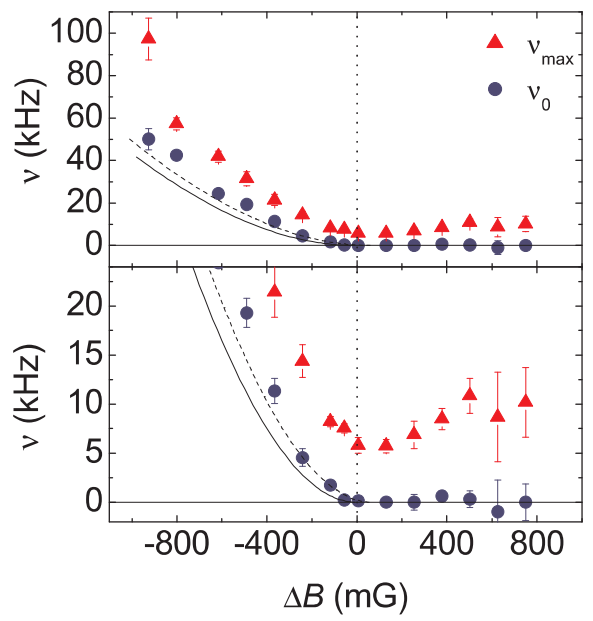

FIG. 3: Threshold frequency $\nu_{0}$ (circles) and frequency of maximum response to perturbation $\nu_{\max }$ (triangles) versus detuning from Feshbach resonance $\Delta B$. The lower graph is a vertical zoom of the upper graph. For comparison, the twobody calculation of the molecule binding energy is shown for a resonance centered at $\Delta B=0$ (solid line) and for a resonance position shifted to $\Delta B=70 \mathrm{mG}$ (dashed line).

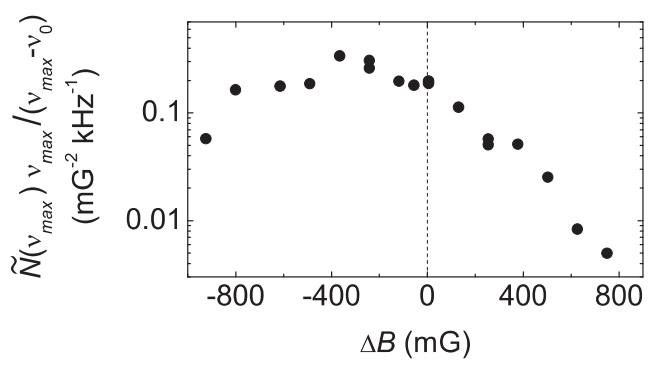

FIG. 4: Dissociation response for various detunings $\Delta B$, plotted as the maximum value of the scaled number of evaporated atoms $\tilde{N}\left(\nu_{\max }\right)$ with the scaling factor $\nu_{\max } /\left(\nu_{\max }-\nu_{0}\right)$ (see text).

On the BCS side we find that the threshold, $\nu_{0}$, is close to zero, suggesting that some atom pairs can be broken by arbitrarily small perturbation energies. We believe that this is a consequence of the density inhomogeneity of the trapped gas. The pairing gap arises from many-body effects and should decrease in the low density regions away from the center of the trap. The frequency location of the maximum signal then provides information about the density dependent pairing gap. We find that the shape of the excitation spectrum is approximately constant on the BCS side and the maximum of the signal occurs at $\nu_{\max }$ between $6 \mathrm{kHz}$ and $11 \mathrm{kHz}$, which is close to twice the Fermi energy. In our experiment we see no significant qualitative change in the spectra when the experiments are performed at slightly higher temperatures above the observed pair condensation temperature. This is consis- 
tent with the expectation that atoms pair up with a pseudogap prior to condensation with a superfluid gap 22. A quantitative theory of the system and its excitations might allow one to extract further information about the pseudogap and/or superfluid gap from the data.

In fig. 4 we plot a measure of the response strength, $\tilde{N}\left(\nu_{\max }\right) \nu_{\max } /\left(\nu_{\max }-\nu_{0}\right)$, versus the $B$-field $\Delta B$, where $\tilde{N}=\left(N_{\text {evap }}-N_{b k g}\right) /\left(b_{0}^{2} t_{\text {pert }}\right)$ is the scaled number of evaporated atoms [33]. Here, we divide $\tilde{N}\left(\nu_{\max }\right)$ by $\left(\nu_{\max }-\nu_{0}\right)$ to account for the heating being proportional to the excess energy above threshold and multiply by $\nu_{\max }$ to account for our frequency dependent modulation amplitude (see eq. 2). We find a strong perturbation signal close to the Feshbach resonance on both sides. The signal greatly decreases for larger positive detunings from the resonance. However, it is still nonzero in a region $\Delta B>500 \mathrm{mG}$ where the measured condensate fraction vanishes. This suggests that in this region noncondensed pairs are present, which would be consistent with a pseudogap theory 22].

In a simple two-body model, in which the interatomic potential is described by a square well and molecules on the BEC-side of the Feshbach resonance are dissociated by periodically modulating the potential depth, we calculate the dissociation rate, $\Gamma\left(\nu_{\text {pert }}\right)$, to be proportional to $\sqrt{h \nu_{\text {pert }}-E_{b}} / h \nu_{\text {pert }}$. However, empirically we find the $\mathrm{BEC}$-side spectra to be more consistent with a rate that goes as $\sqrt{h \nu_{\text {pert }}-E_{b}} /\left(h \nu_{\text {pert }}\right)^{2}$. This suggests that a more sophisticated model is needed.

In conclusion we have introduced a new method for measuring excitation spectra in the BCS-BEC crossover regime. In addition to driving a collective oscillation we find that modulating the interaction strength, by modulating the magnetic field, dissociates fermionic atom pairs. Excitation spectra have been recorded for various interaction strengths, and dissociation thresholds and maxima have been determined. This novel spectroscopy can provide a probe of pseudogap physics and superfluidity at the BCS-BEC crossover.

We thank M. L. Chiofalo, C. H. Greene, C. Ticknor and J. L. Bohn for stimulating discussions. This work was supported by NSF and NIST. C. A. R. acknowledges support from the Hertz Foundation.

* Email: markus.greiner@colorado.edu URL http://jilawww. colorado.edu/ jin/

† Quantum Physics Division, National Institute of Stan- dards and Technology.

[1] D. M. Eagles, Phys. Rev. 186, 456 (1969).

[2] A. J. Leggett, J. Phys. C. (Paris) 41, 7 (1980).

[3] P. Nozieres and S. Schmitt-Rink, J. Low-Temp. Phys. 59, 195 (1985).

[4] M. Randeria, in Bose-Einstein Condensation, edited by A. Griffin, D. W. Snoke, and S. Stringari (Cambridge University, Cambridge, 1995), pp. 355-392.

[5] M. Holland, S. J. J. M. F. Kokkelmans, M. L. Chiofalo, and R. Walser, Phys. Rev. Lett. 87, 120406 (2001).

[6] E. Timmermans, K. Furuya, P. W. Milonni, and A. K. Kerman, Phys. Lett. A 285, 228 (2001).

[7] Y. Ohashi and A. Griffin, Phys. Rev. Lett. 89, 130402 (2002)

[8] M. Greiner, C. A. Regal, and D. S. Jin, Nature 426, 537 (2003).

[9] S. Jochim et al., Science 302, 2101 (2003).

[10] M. W. Zwierlein et al., Phys. Rev. Lett. 91, 250401 (2003).

[11] T. Bourdel et al., cond-mat/0403091.

[12] M. Bartenstein et al., Phys. Rev. Lett. 92, 120401 (2004).

[13] C. A. Regal, M. Greiner, and D. S. Jin, Phys. Rev. Lett. 92, 040403 (2004).

[14] M. W. Zwierlein et al., Phys. Rev. Lett. 92, 120403 (2004).

[15] R. Hulet, private communication.

16] M. Bartenstein et al., Phys. Rev. Lett. 92, 203201 (2004).

[17] J. Kinast et al., Phys. Rev. Lett. 92, 150402 (2004).

[18] W. C. Stwalley, Phys. Rev. Lett. 37, 1628 (1976).

[19] E. Tiesinga, B. J. Verhaar, and H. T. C. Stoof, Phys. Rev. A 47, 4114 (1993).

[20] S. Inouye et al., Nature 392, 151 (1998).

[21] R. B. Diener and T.-L. Ho, cond-mat/0405174.

[22] Q. Chen, J. Stajic, S. Tan, and K. Levin, cond-mat/0404274.

[23] C. A. Regal and D. S. Jin, Phys. Rev. Lett. 90, 230404 (2003).

[24] S. Gupta et al., Science 300, 1723 (2003).

[25] C. A. Regal, C. Ticknor, J. L. Bohn, and D. S. Jin, Nature 424, 47 (2003).

[26] M. A. Baranov, JETP Lett. 70, 396 (1999).

[27] P. Törmä and P. Zoller, Phys. Rev. Lett. 85, 487 (2000).

[28] C. Chin et al., cond-mat/0405632.

[29] B. DeMarco and D. S. Jin, Science 285, 1703 (1999).

[30] Here $T_{F}$ is determined from a Thomas Fermi fit. If $T_{F}$ is instead determined based on the momentum spread, the measured trap frequencies and atom number, we get $T_{F}=h \times 6.0 \pm 0.6 \mathrm{kHz}$, where the uncertainty is statistical. This is in reasonable agreement given the uncertainty in atom number and trap frequency.

[31] C. A. Regal, C. Ticknor, J. L. Bohn, and D. S. Jin, Phys. Rev. Lett. 90, 053201 (2003).

[32] C. Ticknor and J. L. Bohn, private communication.

[33] For $t_{\text {pert }} \geq 10 \mathrm{~ms}$ we apply a correction factor determined experimentally from our measurement of the response versus the perturbation time. 